\title{
The Traveling Salesman Goes Shopping: The Systematic Deviations of Grocery Paths from TSP-Optimality
}

\author{
Sam K. Hui \\ Peter S. Fader \\ Eric T. Bradlow*
}

$1^{\text {st }}$ Submission: November 5, 2006

Revised: April 24, 2007

\footnotetext{
* Sam K. Hui is a doctoral candidate in Marketing, Peter S. Fader is The Frances and Pei-Yuan Chia Professor of Marketing, and Eric T. Bradlow is The K. P. Chao Professor, Professor of Marketing, Statistics, and Education, and Academic Director of The Wharton Small Business Development Center at the Wharton School of the University of Pennsylvania. Corresponding author: Sam K. Hui (kchui@,wharton.upenn.edu). The authors are extremely grateful for the data provided by Sorensen Associates and, in particular, the valuable input and guidance from Herb Sorensen.
} 


\title{
The Traveling Salesman Goes Shopping: The Systematic Deviations of Grocery Paths from TSP-Optimality
}

\begin{abstract}
We examine grocery shopping paths through the lens of the "Traveling Salesman Problem" (TSP), a classic paradigm from the field of operations research. We define the "TSP-optimal" path for each shopper as the shortest path that connects all of his purchases, and we study the systematic deviations seen in his actual behavior. We decompose the length of each observed path into three components: the length of the TSP-optimal path, the additional distance due to order deviation (i.e., not following the TSP-optimal order of category purchases), and the additional distance due to travel deviation (i.e., not following the shortest point-to-point paths). We then explore the relationship between these deviations and purchase behavior. Among other things, our results show a strong relationship between order deviation and basket size, but no association between travel deviation and basket size. Finally, we look at the implications of relaxing three of the rigid assumptions of the TSP by allowing for: (1) varying degrees of "forward-lookingness" across shoppers based on their observed order of purchases, (2) the possibility of unplanned purchases, and (3) the possibility of planned category visits but no resulting purchases.
\end{abstract}




\section{Introduction}

Recently, marketing researchers have shown an increasing interest in studying the instore shopping patterns of consumers and their associated movement paths. With the advent of new technologies, e.g., Radio Frequency Identification (RFID), researchers are equipped with better data to explore in-store movements, adding value to the ubiquitous scanner data analyses that have been pervasive over the past 25 years (Guadagni and Little 1983). For example, Burke (1996) studied consumers' grocery shopping patterns using a virtual (simulated) store; Sorensen (2003) tabulated purchase and time-of-stay statistics at different locations within an actual grocery store; and Larson et al. (2005) categorized grocery paths using a clustering algorithm, and identified 14 different "canonical paths". While these researchers and others have answered many interesting questions and generated useful managerial insights, an important dimension of shopping behavior, namely the efficiency of shoppers, has so far been largely ignored. Do shoppers tend to be somewhat "optimal" in their shopping patterns, and in what ways do they systematically depart from "optimality"? These are questions that are of interest not only to academicians, but also to retailers who may wish to understand how consumers tend to navigate within their stores as they gather their purchases.

At the heart of our research is the "Traveling Salesman Problem" (TSP), a classic paradigm in operations research. In the TSP, the salesman has to visit a number of cities before returning to her original starting point. The objective is to choose her order of visitation in order to minimize her travel distance while visiting all the required cities. In this respect, the TSP closely resembles the problem faced by a hypothetical grocery shopper who plans to purchase a certain list of items in the grocery store. For the shopper, the TSP "solution" refers to the shortest path that connects the entrance, all the products that she purchases, and the checkout counter; 
henceforth, we refer to this path as the "TSP-optimal" path. Once the TSP-optimal path is identified, we can then compare it to each shopper's observed behavior and document the systematic departures that may exist.

To follow the TSP-optimal path, the grocery shopper needs to solve two separate yet interrelated problems. First, he needs to identify the optimal order to pick up his products. Then, given this order, he has to determine the shortest point-to-point path to move from one product location to the next. Actual shopper behavior, however, may depart from TSP-optimality in either or both of these ways.

First, the shopper may follow a different algorithm when deciding on an order of visitation. For instance, he may follow a "greedy" (1-step look-ahead) algorithm (Cormen et al. 2001), where he always moves towards the closest unpurchased product. More generally, he may follow a $k$-step look-ahead algorithm (Cormen et al. 2001; Camerer et al. 2004), where he plans ahead $k$ steps before deciding which product to head towards next. Since the TSP can be viewed as a look-ahead algorithm with $k=\infty$, these other algorithms generally lead to longer shopping paths. In this research, we define this type of departure from TSP-optimality as "order deviation" (i.e., following a sub-optimal order of purchases), and we will use it to make inferences about consumers' look-ahead propensities based solely on their observed purchases and paths.

Second, the shopper may not follow the shortest point-to-point travel path when he moves from one product to the next, given the order of purchases he has chosen. He may deviate from the shortest point-to-point path for various reasons, such as: (1) he may not have perfect knowledge about the store's layout, (2) he may be distracted by in-store merchandising activities, or (3) he may be shopping around for purely hedonic reasons. Regardless of the specific reason(s), we define this source of departure from TSP-optimality as "travel deviation." 
While in some contexts, the TSP may be viewed as a descriptive model of the actual optimal behavior (e.g., Eilon et al. 1971; Lenstra and Rinnooy Kan 1975), that is not the goal of this paper. Instead, we take TSP-optimality as a frame of reference against which we compare observed behavior. Thus, we do not claim that the deviations in the in-store shopping context from the TSP solution are necessarily inefficient. Shoppers may enter with different goals and with different levels of knowledge about the store. Thus we stress that, in this paper, we do not associate any notion of "goodness" or "badness" to the TSP-optimal solution and the corresponding deviations. Whether these deviations are advantageous or disadvantageous from the standpoint of the consumer or the retailer is an important issue for future research (which we discuss in Section 8), when additional data from a field study may become available.

We note that our research is in the same spirit as other papers in marketing/economics that have compared observed behavior to a normative paradigm. For example, Camerer et al. (2004) analyzed people's behaviors in economic games and compared them with the normative solutions prescribed by the Nash equilibrium. Likewise, Meyer and Assuncao (1990) analyzed consumers' stockpiling strategies and documented the contexts in which consumers tend to underbuy or overbuy compared to their optimal solutions, calculated from sequential decision theory. In both cases, researchers took a well-established optimality paradigm, then analyzed and explained the systematic deviations from optimality that they observed in actual behavior. Other related papers in other contexts include Houser, Keane, and McCabe (2004), Hutchinson and Meyer (1994), Neslin and Greenhalgh (1983), and Seale and Rapoport (2000).

The remainder of this paper is organized as follows. In the next section, we develop the theoretical background of our research by reviewing the relevant literature for the TSP. Section 3 describes the decomposition of each observed path into TSP-optimality, order deviation, and 
travel deviation. In Section 4, we provide a brief overview of our dataset and describe relevant summary statistics. In Section 5, we categorize and analyze shoppers based on their order and travel deviations and relate them to various aspects of shopping and purchase behavior. Then, in Section 6, we explore shoppers' "look-ahead" behavior, i.e., we infer the $k$-step forward-looking behavior of consumers that is most consistent with their observed path data. Section 7 describes two additional sensitivity analyses that relax the TSP assumption that shoppers utilize a fixed shopping list. Finally, Section 8 concludes with additional discussion and directions for future research.

\section{Relevant literature}

The TSP has been widely applied in many different areas in operations research and engineering. Many real-life optimization problems can be naturally transformed into and solved as a TSP problem. For example, researchers have applied the TSP to address job sequencing, i.e., deriving the optimal order of performing a list of tasks on a machine (Bellmore and Nemhauser 1968; Gilmore and Gomory 1964). Researchers have also used TSP methods to assign traffic routes to various destinations in order to minimize aggregate travel time (e.g., Eilon et al. 1971; Lenstra and Rinnooy Kan 1975). In engineering, the TSP has been applied to tackle various problems such as designing interfaces for computer wiring (Lenstra and Rinnooy Kan 1975), and planning the motion of robot arms (Chalasani et al. 1996). While these examples are somewhat different from the grocery setting that we study (because the goals in these examples are more well defined), they nonetheless offer some guidelines to how the TSP can be applied in marketing settings. Interested readers are encouraged to see Lawler (1985) for a more detailed introduction to the TSP and its applications. 
Despite its popularity in other domains, the TSP is rarely mentioned or utilized in the marketing literature. For instance, we are unaware of any applications of the TSP ever published in the Journal of Marketing Research or Marketing Science. This may be due to the lack of available data suitable for modeling using the TSP framework. In the future, however, we may see more use of the TSP in marketing due to the increasing availability of various forms of path data in general (e.g., Hui et al. 2007b).

Another stream of research, in cognitive psychology, studies how people perform TSPtype tasks in laboratory settings. Polivanova (1974) studied subjects' performance when simple (e.g., 4, 6, 8-node) TSPs were presented in a visual versus a textual format, and found that subjects' performance improves significantly when a visual format is used. Her pioneering study was followed up by several other psychologists. For instance, MacGregor and Ormerod (1996) studied how people's performance changed with the complexity of the TSP problem, as measured by the total number of "non-boundary" points. MacGregor et al. (1999) further studied the effects of spatial and contextual factors on human TSP performance. Vickers et al. (2001) ran a series of experiments with visually-presented TSPs and analyzed how the process of path formation is related to the structure of the spatial layout and individual-based differences. MacGregor et al. (2000) proposed a model of how subjects solve (visually presented) TSP problems.

In all of these cases, researchers have focused primarily on people's performance in visually-presented TSP problems in experimental settings. Thus, our project contributes to this stream of research by studying to what degree people follow TSP-like behavior in an actual grocery store. In the grocery setting, the TSP problem is not presented directly to the shopper in a two-dimensional layout; instead, the shopper is immersed in an real shopping environment and 
(implicitly) deals with a TSP-type problem as he interacts with the spatial environment. Thus, beyond our primary focus on grocery shopping behavior, our work adds new insights to the existing studies of how people perform in TSP problems more generally.

Two other streams of research that relate to our study include trip chaining, which studies how people carry out a set of errands that take place in different locations (e.g., Adler and BenAkiva 1979; Dellaert et al. 1998; Thill and Thomas 1991), and wayfinding, which studies how people orient themselves in a spatial environment and navigate through space to reach their destinations (e.g., Dogu and Erkip 2000; Golledge 1999; Titus and Everett 1996). The focus of this research, however, is different from the central issues in these research areas; rather than trying to develop a descriptive model, we are instead using the TSP as a logical benchmark to allow us to better understand the kinds of behavioral patterns that actually take place in a grocery store.

\section{Deviation decomposition}

In section 3.1, we define the TSP-optimal path for each grocery shopper. Using the parallel structure between our problem and the TSP, we apply the solution methods developed for the TSP to obtain the TSP-optimal path for each shopper. In Section 3.2, we relax the assumption of unbounded rationality on the part of the consumer, and study the optimal path under a $k$-step look-ahead algorithm. In section 3.3, we decompose the departure of each shopper's observed path from its TSP-optimal counterpart into two components: order deviation, which captures the deviation due to picking up purchases in a different order than that suggested by the TSP, and travel deviation, which results because shoppers may not follow the shortest point-to-point travel path when going from one location to the next.

\subsection{TSP-optimal paths}


We define the TSP-optimal shopping path as the shortest path (in terms of total travel distance) that starts at the entrance, connects all of the observed purchases, and ends at the checkout. In the TSP, the optimal path is based on four behavioral assumptions, which we translate into the grocery context as follows:

(i) The shopper comes into the store with a pre-determined shopping list.

(ii) The shopper has perfect knowledge of the store layout and product locations.

(iii) The shopper aims to minimize his in-store travel distance (i.e., the optimal solution does not apply to "hedonic" browsers).

(iv) The shopper has enough cognitive capacity to solve highly complex optimization problems in his mind.

Although we do not believe that any or all of these assumptions will necessarily hold in reality, they collectively provide a natural frame of reference to which we can compare shoppers' actual behavior. Further, these assumptions can be relaxed to generate different notions of "optimality". For instance, we generalize (iii) and (iv) in Section 3.2 and derive the "optimal" path under a $k$-step look ahead rule. We also study the extent to which our results will be changed by relaxing assumption (i) in a sensitivity analysis in Section 7. Likewise, one can consider relaxing (ii), in which case the problem will be akin to an "online TSP" problem, a current research area in operations research (Ausiello et al. 2001). ${ }^{1}$

Many methods have been proposed to solve the TSP, e.g., cutting planes method, branch and bound, and genetic algorithms (see Lawler 1985). In this paper, we use two different algorithms that are commonly employed to solve the TSP: exhaustive search and simulated annealing. For paths with 10 or fewer purchase locations, we identify the optimal path by

\footnotetext{
${ }^{1}$ In the online TSP problem, not all the information about the spatial structure is revealed to the shopper at the beginning; instead, shoppers learn about the spatial structure over time, governed by an "information schedule" (Ausiello et al. 2001).
} 
exhaustively searching through all possible permutations to find the order with the minimum travel distance. For paths with more than 10 purchase locations, the number of possible permutations is too large (and thus computationally infeasible) to conduct an exhaustive search. For instance, a path with 11 purchase locations will generate $11 !=39,916,800$ permutations. In those cases, we use a simulated annealing algorithm (Goffe et al. 1994) to stochastically search for the globally optimal solution. To ensure that our algorithm is able to locate the global optimum, we use a very slow exponential cooling schedule and run our algorithm multiple times from different starting points. In addition, we also validated our annealing algorithm on paths where we already know the optimal solution (i.e., paths with 10 or fewer purchase locations), confirming its accuracy. The implementation details are included in Appendix 1.

\subsection{Optimal paths under $k$-step look ahead}

In this section, we describe the extension of the TSP in which we relax the assumption that each shopper is unboundedly rational. Instead, we compute what the "optimal" path will be for a shopper who is able to look ahead only $k$-steps ${ }^{2}$ (i.e., his next $k$ purchases) before deciding on his next step (purchase). More specifically, we define a $k$-step look-ahead algorithm as follows: A shopper with $k$-step look ahead will consider the total distance of the next $k$ steps and find the permutation with the minimum length. ${ }^{3}$ He then takes the first step of this optimal order (i.e., moves to the location of the first item to be purchased) and begins the process again. The TSP-optimal solution can be viewed as being equivalent to a look-ahead algorithm with $k=\infty$. Another special case of the $k$-step look-ahead algorithm is the "greedy" algorithm, with $k=1$. In

\footnotetext{
${ }^{2}$ Throughout the entire paper, we use the word "step" to denote a purchase. We use the phrase " $k$-step look ahead" instead of the (perhaps more appropriate) " $k$-purchase look ahead" to be consistent with the terminology used in the optimization literature (e.g., Cormen et al. 2001).

${ }^{3}$ If $k$ is larger than the total number of remaining purchases, the shopper does not need to re-compute the optimal path after each purchase, because of the principle of optimality. We thank an anonymous reviewer for pointing out this issue.
} 
this case, a shopper will always head towards the unpurchased item that is closest to his current location.

We illustrate the $k$-step look-ahead algorithm in the following example. In order to focus our attention on illustrating the algorithm, we abstract away from the context of the grocery store and instead consider the stylized geometry as shown in Figure 1. Assume that the shopper wants to purchase three products, which we denote as A, B, and C. The distances between the entrance, A, B, C, and the checkout are shown in Table 1.

[Insert Figure 1 about here]

[Insert Table 1 about here]

We compute the optimal path under $k=1$ ("greedy" algorithm) up to $k=4$ (which is TSPoptimal in this case). Under $k=1$, the shopper first considers whether to go to A, B, or C for his first step. To do so, he considers only the immediate next step from his current location, i.e., the distance between the entrance to A, B, and C. Since C is closest to the entrance (4.91 units), he takes $\mathrm{C}$ as his first step. Then, at $\mathrm{C}$, he considers whether to go to $\mathrm{A}$ or $\mathrm{B}$, again based on the distance from $\mathrm{C}$ (his current location) to $\mathrm{A}$ and $\mathrm{B}$. He picks $\mathrm{B}$ because it is closer to $\mathrm{C}$ than $\mathrm{A}$. Thus, his order of visitation, under 1-step look ahead, is $\mathrm{C} \rightarrow \mathrm{B} \rightarrow \mathrm{A}$, for a total distance of 21.63.

When $k=2$, the shopper considers two steps ahead when making a visit decision. Starting at the entrance, he considers the total distance of his first two steps. There are six permutations he needs to consider, namely $\mathrm{AB}, \mathrm{AC}, \mathrm{BA}, \mathrm{BC}, \mathrm{CA}$, and $\mathrm{CB}$. To decide among these options, he calculates the distance from the entrance to his first move plus the distance between the first and second move. For each of the six options, the total distance (for next two moves) are shown in Table 2 .

[Insert Table 2 about here] 
We see that the sequence minimizing total distance (for the first two steps) is BA. Thus, the shopper chooses B as his next location to visit. At B, he has to choose between going to A or C next (i.e., once reaching a location, the $k$-step optimization process begins anew). He considers two possible sequences (for his next two steps): AC or CA. Again, he evaluates the total distance of his next two steps and finds that the sequence AC gives a shorter distance. Thus, he decides that his next location to visit is A. Thus, his order of visitation, under 2-step look ahead is $\mathrm{B} \rightarrow \mathrm{A} \rightarrow \mathrm{C}$. Using a similar argument as above, we derive that the order of visitation, under 3-step look ahead, is $\mathrm{C} \rightarrow \mathrm{A} \rightarrow \mathrm{B}$.

When $k=4$, the shopper considers all orders of visitation and calculates the total trip distance at the entrance, before choosing a sequence of visitation. Thus, he calculates all the distances associated with each visit order, as shown in Table 3. He chooses the order $A \rightarrow B \rightarrow C$ because it offers the shortest distance for the entire trip.

\section{[Insert Table 3 about here]}

Before generalizing from this stylized example, it is useful to acknowledge three caveats about its results: (1) In this case, there is a monotone relationship between $k$ and the total path length; however, this may not be necessarily true in general. (2) We see that the biggest decrease in path length occurs between $k=3$ and $k=4$ (i.e., the final step towards TSP-optimality); however, this does not necessarily hold in general. (3) We observed a different path for each value of $k$, but this need not be true in most cases. Caveats aside, this simple example demonstrates how different look-ahead algorithms may lead to different orders of visitation, and we will pursue this issue in more detail in Section 6.

\subsection{Order and travel deviations}


Once we identify the TSP-optimal solution, each observed path can be decomposed based on its departure from the path suggested by the TSP. As we mentioned earlier, two types of deviations are considered, which we call order deviation and travel deviation. We illustrate each of these deviations using Figure 2 as an example.

\section{[Insert Figure 2 about here]}

Order deviation refers to the excess distance resulting from picking up products in a different order than that suggested by the TSP solution. For example, as can be seen in Figure 2, the TSP-optimal order is $\mathrm{B} \rightarrow \mathrm{A} \rightarrow \mathrm{C}$, with a total travel distance of $3+3=6$ units. The observed shopping order, $\mathrm{A} \rightarrow \mathrm{C} \rightarrow \mathrm{B}$ results in a longer travel distance $(5+3=8)$, assuming that he takes the shortest point-to-point paths when traveling between two locations. We define the difference between the travel distance of the optimal order and the observed order as order deviation. Thus, the order deviation here is $8-6=2$ units.

The second source of deviation, travel deviation, arises when a shopper does not follow the shortest point-to-point route as he travels from one location to the next. In Figure 2, for instance, although the shortest path from $\mathrm{B}$ to $\mathrm{C}$ requires 5 units of travel distance, the shopper takes a more indirect path that requires 7 units of travel distance. The difference, 2 units, is the amount of travel deviation the shopper incurs when he travels from B to C. Likewise, he incurs 4 $-3=1$ unit of travel deviation when he travels from C to A. Thus, there is a total of 3 units of travel deviation.

To summarize, each observed path can be decomposed into three components: the TSPoptimal path, order deviation, and travel deviation. Adding these three components together equals the total distance traveled. In our example above, the decomposition can be described by the following equation: 


$$
\begin{aligned}
& \text { Observed Path = TSP-Optimal Path + Order Deviation + Travel Deviation } \\
& 11=6+2+3
\end{aligned}
$$

This decomposition represents the heart of our analysis and generates a variety of questions (which we will address). For instance, this stylized example results in distances covering 55\%, $18 \%$, and $27 \%$ of the trip for TSP-optimality, order deviation, and travel deviation, respectively. But what will these proportions be in reality and how much will they vary across trips? Will one component dominate the others? And how will this decomposition relate to other trip characteristics such as the number of items purchased on each trip? In the next section we describe the data used in our analysis, and then we proceed to answer these (and other) questions.

\section{Data}

While TSP-type problems have been of great interest to academicians and practitioners alike, the paucity of available in-store data has made such research difficult if not impossible in most marketing contexts. Recent technologies and advances, however, now enable an entirely new set of insights. In this research, we analyzed a unique and novel dataset from a large supermarket in the eastern United States that contains consumers' shopping path data together with their purchases (as recorded from scanner data). We obtained our data from Sorensen Associates, an in-store research company that tracks shoppers' movement using its proprietary PathTracker ${ }^{\circledR}$ system based on Radio Frequency Identification (RFID) technology (Sorensen 2003). A small RFID tag is affixed under each shopping cart, and emits a uniquely coded signal every five seconds; this signal is then picked up by an array of antennae located throughout the store which can pinpoint the precise location of the shopping cart over time (Sorensen 2003).

After performing the data cleaning and preparation procedure described more fully in Hui et al. (2007a) and outlined in Appendix 2, our dataset contains a total of 993 shopping paths and 
their corresponding purchase records. ${ }^{4}$ The procedure in Hui et al. (2007a) allows us to discretize the grocery store into a graph with 96 nodes, hence making each cart movement a selection among a finite set of edges. The division of the grocery store into zones is shown graphically in Figure 3 and Figure 4. We refer the interested reader to Appendix 2 for more details of our data preparation procedure.

[Insert Figure 3 about here]

[Insert Figure 4 about here]

For each path, we extract a number of key summary statistics (shown in Table 4) that jointly capture shoppers' movement and purchases. As mentioned earlier, we will relate these measures to the degree of TSP-optimality and the associated deviations across the set of trips. These statistics include the total number of product categories purchased out of a total of 116 categories, total path distance traveled in the store, the number of unique zones visited (out of the aforementioned 96 zones), and the number of unique aisles that each shopper entered and traversed. Table 5 lists the top ten categories purchased based on the proportion of shoppers who made at least one purchase in each category, another metric of interest. While we acknowledge that it would be worthwhile to consider financial metrics such as the dollar amount spent by each shopper or the margin that retailers extract from each category, this information is not available in our dataset. Thus, we focus only on category purchase incidence.

[Insert Table 4 about here]

[Insert Table 5 about here]

\footnotetext{
${ }^{4}$ Because of the nature of the PathTracker ${ }^{\circledR}$ data collection method, trips that do not use a cart are not considered, which constitutes a substantial number. Thus, our results may not be representative of overall grocery shopping. In particular, we would expect a much higher degree of TSP-optimality for the many 1-2 item trips that actually exist.
} 
Many interesting research questions can be raised by looking at the relationship between the variables shown in Tables 4 and 5 and the deviation measures identified in Section 3. For instance, will longer trips be associated with higher or lower order deviations? On the one hand, longer trips may be more organized and less chaotic than shorter trips; yet on the other hand, there are more opportunities for choosing an order of visitation that is different from the TSP solution. Another interesting issue is whether/how category purchase incidence is in any way related to order/travel deviations. For example, do shoppers who are more TSP-optimal tend to shop disproportionately in certain categories? What is the relationship between order/travel deviations and the size of market baskets? We study these and other questions in detail in our analyses in Sections 5 and 6.

\section{Decomposition analysis}

The fractional distances associated with the TSP-optimal path, order deviation, and travel deviation, for each of the 993 paths, are shown graphically in the triangle plot in Figure 5, and the associated summary statistics are contained in Table 6 . The triangle plot allows us to easily visualize the relationship among three variables that sum to 1 . This figure yields several immediate insights. First, there is a great deal of variability in the degree of TSP-optimality across the 993 trips. The percentage of TSP-optimality ranges from a low of approximately $5 \%$ to a high around $95 \%$, with an average of about $28 \%$ of the total travel distance.

In contrast to the high degree of variability in the percentage of TSP-optimality, the extent of order deviation is quite limited - never exceeding $20 \%$. This suggests that shoppers in general choose an order for their purchases that is fairly close or the same as the order suggested by the TSP solution. 
Most of the trips lie in the lower right corner of Figure 5, indicating that travel deviation accounts for a large portion of the travel distance in the majority of grocery trips. So while the order of purchases is close to TSP-optimal, shoppers spend a large portion of their in-store trip not following the shortest point-to-point travel paths. One potential reason (among many others) for this large deviation is that shoppers may deliberately plan to visit some product categories to see whether promotions are available, but may not necessarily purchase from those categories. We investigate this issue in more detail in Section 7.

[Insert Figure 5 about here]

[Insert Table 6 about here]

To get a better idea about the relationship between these measures of deviation and the characteristics of shopping paths mentioned earlier, we divide the 993 trips into four different clusters $(\mathrm{H}=\mathrm{high} ; \mathrm{L}=\mathrm{low})$ based on a median split along each deviation dimension. The summary statistics for each group are shown in Table 7, along with relevant visit and purchase characteristics, as described in aggregate in Table $4 .^{5}$

The first, and most obvious, contrast is between group 1 (low on both deviation dimensions) versus group 4 (high on both). It should come as no surprise that shoppers who exhibit most deviations from the TSP solution tend to visit more zones, which means entering (and traversing) more aisles. It is not as obvious, a priori, that these shoppers will also buy more products, but the difference in basket size (as well as each of the other metrics in Table 7) is large and highly significant $(\mathrm{p}<.001)$.

\footnotetext{
${ }^{5}$ Note that our data are strictly correlational, and we are therefore cautious about drawing causal conclusions from them.
} 
A more illuminating contrast is between the two intermediate groups. In comparing group 2 to group 3, we see that order deviation tends to be more influential than travel deviation in generating long trips with more aisles visits/traverses and larger baskets of purchased items. But a closer look at these two groups reveals some interesting differences that reflect the impact of order vs. travel deviation. For instance, while the average basket size is over $50 \%$ greater for group 2 vs. group 3, the mean number of zones visited is barely $10 \%$ larger. The latter difference is still statistically significant $(p=.003)$, but it is indicative of the notion that the shoppers who exhibit a lot of travel deviation are visiting an excessive number of zones relative to the number of items they purchase.

When we aggregate the data in Table 7 to look at each of the deviation dimensions by itself, we see another interesting trend involving basket size. Specifically, mean basket size is far smaller for the groups with low order deviation, i.e., groups $1 \& 3$ (mean $=5.1)$ compared to those with high order deviation, i.e., groups $2 \& 4$ (mean $=9.1, \mathrm{p}<.001)$. This observation is consistent with MacGregor and Ormerod (1996), who found that people's performance in TSP problems generally worsens (i.e., more order deviation in our context) when they are given more locations to visit. But when we aggregate along travel deviation (groups $1 \& 2$ vs. $3 \& 4$ ) we see no difference in basket size (means of 7.0 and 7.2, respectively, $p=.35$ ). Thus, while travel deviation accounts for a large portion of most trips, order deviation has a much stronger association with purchasing behavior.

\section{[Insert Table 7 about here]}

We are also interested in identifying which product categories are most strongly associated with each of the four clusters. To perform this analysis in a reasonable manner, we must normalize for the differences in basket size. To do so, we compute the number of 
purchases of each category for each group, and divide this by the total basket size of each group; these proportions are then compared across groups. Table 8 displays the product categories that are significantly (at $\mathrm{p}<.05$ level) over-represented in at least one group. We find that produce (e.g., fruits and vegetables), deli products (e.g., cheese/milk), and pre-packaged products tend to be associated with the groups that have low levels of order deviation; they seem to correspond to a well-organized shopping trip with a specific purpose, e.g., a shopper who brings a shopping list to shop for frequently purchased items. Along these lines, note that four of the ten most frequently purchased categories (in Table 5) are overrepresented in group 1. On the other hand, less frequently purchased household products are associated with higher order deviations. These purchases may correspond to a more impulsive shopping trip; on such trips, the shoppers may be shopping casually and choosing categories as they go along, without much concern about planning their trip. This results in a longer shopping path, and a seemingly haphazard path between purchases. Alternatively, it is also possible that these are "price shoppers" who are looking for promotions, an issue that we will study in more detail in Section 7. A further explanation is that the location of less frequently purchased items is more unknown, leading to greater travel deviation. Future research involving the aforementioned online-TSP problem can address this hypothesis.

[Insert Table 8 about here]

\section{Inferring the degree of look-ahead behavior}

The analysis so far suggests that order deviation is a much better discriminator of the shopping behavior across different types of consumers compared to travel deviation. In some sense, travel deviation represents an ambient level of "noise" that affects virtually all shopping paths. Thus, in this section, we focus on using only shoppers' order of purchases to estimate the 
number of "look-ahead" steps that they consider. As discussed in Section 3.2, a shopper with $k$ step look ahead will consider all possible permutations of the next $k$ steps (purchases) and find the permutation with the minimum length, then take his next step (purchase) based on this calculation.

Since we only observe one path for each shopper (our dataset is purely cross-sectional), we can only estimate the value of $k$ through a set of heuristic calculations. Thus, our results are exploratory, rather than conclusive, in nature; we encourage other researchers to carry out more careful laboratory experiments to replicate/verify our results from this field data. To assess the value of $k$, we propose a two-step heuristic: first, we consider only order deviation (ignoring travel deviations) and compare the path lengths with those generated using a 1-step look-ahead ("greedy") algorithm. We divide shoppers into two groups depending on whether their observed order generates a longer or shorter distance than the order associated with 1-step look ahead. Then, we separately estimate $k$ for each group.

We find that in our data, $489(49.2 \%)$ of the shoppers follow an order that generates paths that are shorter or equal to that suggested by the 1-step look ahead; we label them as group A. The remaining 504 shoppers $(50.8 \%)$ consists of shoppers who follow an order that generates paths that are longer than that suggested by 1-step look ahead, and are labeled as group B. For this group, the value of $k$ is inferred to be less than or equal to 1 .

Before estimating $k$ for each shopper within these groups, we first compare the two groups in aggregate using the same purchase and visit statistics as before. Table 9 offers some insights about how purchase/visit behavior are related to shopper's degree of "forwardlookingness." We observe that there is almost no difference between the high- $k$ and low- $k$ groups in terms of travel deviation, once again confirming the notion that the degree of travel 
deviation is not strongly associated with look-ahead tendencies. Second, the low- $k$ group tends to visit more zones and has a higher average basket size than the high- $k$ group $(\mathrm{p}<.01)$. This means that longer trips are associated with a lesser degree of look-ahead behavior. Naturally, less forward-looking shoppers tend to enter and traverse more aisles $(\mathrm{p}<.01)$.

[Insert Table 9 about here]

Similar to our analysis in Section 5, we look at the categories that are purchased by each of the two groups and note the systematic differences, after normalizing for different basket sizes. Table 10 shows the product categories that are significantly over-represented (at a level of $\mathrm{p}<.05$ ) in one of the groups. We see that the produce and tobacco categories are over-represented in the "high- $k$ " group. On the other hand, canned, ready-to-eat, and frozen food, among other products, tend to be over-represented in the "low- $k$ " group. This indicates that on average, shoppers who purchase prepared food products are generally less forward-looking than other shoppers when they construct their shopping paths. At the surface, inferences like these may seem to be of only tangentially managerial relevance; however, through a field study, a retailer may attempt to influence $k$ through advertising, in-store signage, etc., and note whether profits and sales are associated with various look-ahead patterns, and potentially develop the measure $k$ into a useful managerial tool. We return to this issue in Section 8.

[Insert Table 10 about here]

We now proceed to estimate $k$ for each individual shopper. For each path in group A, we calculate the "optimal" path calculated using a $k$-step look-ahead rule (using the procedure in Section 3.2), and record the optimal path length associated with each $k$. Then we assign each shopper to the value of $k$ that minimizes the absolute difference between the distance associated with $k$ and the distance associated with the person's observed order. That is, each shopper is 
assigned a value of $k=k^{*}$ so that their observed order of visitation is most consistent with the corresponding optimal path calculated using a $k^{*}$-step look-ahead algorithm.

For shoppers in group B, they follow an order that is less efficient than that suggested by 1-step look ahead, so the algorithm above can not be directly applied to them. To profile this group of shoppers, we create an "optimality index" $p$ between 0 and 1 . The optimality index $p$ is defined such that an index of 1 indicates that the 1-step look-ahead rule is always followed (i.e., $p=1 \Leftrightarrow k=1$ ), while an index of 0 indicates that a random rule (or 0 -step look ahead) is followed, i.e., the shopper randomly chooses his next location at every step $(p=0 \Leftrightarrow k=0)$. Heuristically, we interpret the intermediate values of the optimality index as the fractional amount of look ahead a person follows, relative to the 1-step look-ahead rule. The higher the value of $p$, the more closely a shopper follows the 1-step look-ahead rule. We estimate the value of $p$ using a likelihood-based procedure; the details are included in Appendix 3.

The resulting estimates of $p$ for group B are shown in Figure 6. The median value of $p$ for these shoppers is 0.48 , while the corresponding mean is 0.47 .

\section{[Insert Figure 6 about here]}

We then combine groups A and B and display the complete distribution of $k$ in Figure 7. The overall median value of $k$ is 0.99 , while the overall mean of $k$ is 1.36 . The mean and median values of $k$ inferred from our data are consistent with the agent-based simulation literature (e.g., Schelhorn et al. 1999), where researchers commonly assume that shoppers follow a 1-step look ahead rule. Our estimates are also quite consistent with the results obtained by Camerer et al. (2004), who analyzed a series of economic games and find that people's average number of lookahead steps is around 1.5. This linkage to the other studies may be entirely coincidental, but it is undoubtedly worthwhile to investigate these patterns in greater depth in future research. 
[Insert Figure 7 about here]

\section{Sensitivity Analysis}

So far, our decomposition analysis has been based on the universally employed TSP assumption that shoppers come to the store with a fixed shopping list, or at least a clear idea of what they plan to buy during the trip. This assumption is unlikely to hold in practice. First, some categories purchases are unplanned; i.e., the shopper may pick up and purchase some items as she passes them, without previously planning to buy from those categories when she entered the store. Second, a shopper may plan to visit some categories to check for promotions, but may not purchase from them if a suitable deal is not available. In both cases, the shopper may exhibit departures from the TSP-optimal path because the set of categories that the consumer planned to visit is different from the set that she purchased. To study the extent to which these violations of the "fixed shopping list" assumption affect our results, we conducted two sets of sensitivity analyses with respect to each of two aforementioned scenarios.

In the first sensitivity analysis, we randomly assign, for each trip, some of the purchases to be "unplanned," then we re-compute the "triangle" decomposition based on the reduced set of categories. Table 11 below shows the results when $10 \%, 20 \%, 30 \%, 40 \%$, and $50 \%$ of observed purchases are treated as unplanned. Since the assignment algorithm is stochastic, we repeat this procedure ten times and report the average across those runs.

\section{[Insert Table 11 about here]}

We find that by allowing some of the categories to be unplanned, the TSP-optimal portion of each path is reduced; this is expected because while the total observed distance is unchanged, the optimal distance (i.e., the minimum distance that the consumer needs to travel in order to complete his planned purchases) is reduced, thus reducing the extent of TSP-optimality 
(from $27.5 \%$ under the original no-unplanned purchase scenario to $22.1 \%$ when $50 \%$ of purchases are treated as unplanned). Because the movements towards unplanned purchases are treated as deviations from the main path, the fraction assigned to travel deviation therefore increases (from $69.4 \%$ to $76.6 \%$ as we go from $0 \%$ to $50 \%$ unplanned purchases). On the other hand, the extent of order deviation decreases (from 3.1\% to 1.3\%), due to the removal of these unplanned purchases from the original shopping list. While all of these changes are quite smooth and monotonic, we don't see such a clear pattern in our estimates of the "forward-lookingness" indicator, $k$. These estimates don't change much as we increase the degree of unplanned purchases, but there is some indication of an inverted-U pattern, peaking at $20 \%$. We refrain from drawing any definitive conclusions from this initial evidence, but instead we call for more research into this issue to better understand this effect.

In the second sensitivity analysis, we randomly add $m$ categories to each consumers' shopping basket to represent categories that she initially chose to visit (i.e., before entering the store), but did not purchase from. They may represent categories that consumers regularly check for promotions. We restrict the categories to be added to ones that are located in store areas that the consumer actually passed during her trip. Again, since the assignment algorithm is stochastic, we repeat each scenario ten times and report the average across ten runs below for $m=0,1,2, \ldots$ 5 (the latter being a very large number compared to the average basket size). The results are shown in Table 12.

[Insert Table 12 about here]

We see that by allowing some category visits to be planned but not purchased, the fraction of the trip accounted for by travel deviation decreases with $m$ (i.e., from $69.4 \%$ to $58.0 \%$ as $m$ rises from 0 to 5). This is because a portion of the travel deviation is now treated as 
planned visitation of certain categories (e.g., to check for promotions). In addition, since the total observed trip length remains the same while the optimal path becomes longer (to cover the categories that the consumer plans to visit), the fraction of TSP-optimality goes up (from $27.5 \%$ to $36.1 \%)$. Finally, the fraction of order deviation goes up due to the inclusions of these additional product categories in the consumers' shopping list (from 3.1\% to 5.9\%). The estimates for the average values of $k$ are fairly stable across the different scenarios.

The above sensitivity analyses are valuable in at least three respects: (i) They show that our results (especially the estimate for average $k$ ) are reasonably invariant with respect to violations of the TSP assumptions, namely unplanned purchases, and category visits that are planned but do not result in purchases; (ii) they allow us to explore the directionality and magnitude of how our decomposition results will be affected when the "fixed shopping list" assumption is violated; and (iii) they show that at least part of the travel deviation can be attributed to consumer search behavior.

\section{Discussion and conclusions}

In this research, we analyzed grocery shopping paths using the Traveling Salesman Problem (TSP) as a frame of reference. We decomposed the systematic deviations between the observed path and the corresponding solution of the TSP problem into two components: order deviation and travel deviation, and used these measures as a basis to cluster shoppers and study their purchase behavior.

At the heart of this decomposition analysis is a triangle plot (Figure 5), which offers a mixed answer to the central question, "how TSP-optimal are grocery trips?" On one hand, relatively few of them have a degree of TSP-optimality that captures over $50 \%$ of their travel 
distance; but on the other hand, the degree of order deviation is very low in every case - never exceeding $20 \%$ of the travel distance. Thus shoppers tend to pick up their purchased products in an order close to that suggested by the TSP, but tend to depart from the shortest point-to-point path (i.e., travel deviations) as they move through the store.

Although we do not see a great deal of order deviation, this does not imply that shoppers are looking ahead many steps as they make their purchases in the store. In contrast, many of them have a "look-ahead" index of $k=1$, suggesting that they are using a relatively naïve "greedy algorithm" to chart their path. Nevertheless, this is still higher than a quasi-random shopper (with $k<1$ ), which characterizes roughly half of the trips we have observed.

Both analyses reveal consistent patterns about the interrelationship between order deviation and other characteristics of the trip. Specifically, trips with high order deviation (which corresponds to low $k$ ) tend to be longer trips with a greater number of product categories purchased. Travel deviation is also associated with longer trips, but has no association with the overall basket size.

From a managerial standpoint, these results have significant face validity yet we believe that they were not obvious a priori. Practitioners are keenly interested in understanding these notions of "efficiency," but there is not a clear understanding of whether these deviations (and which type of deviation, to be more specific), are desirable or undesirable from a manager's perspective. On the one hand, deviations from the TSP solution give the shopper additional opportunities to see (and perhaps buy) more products - this might be a good outcome for the retailer. But on the other hand, part of the deviations may be due to confusing product placement and poor store layout - which will create dissatisfaction among shoppers. Our current dataset, by itself, cannot shed much light on this dichotomy, but it would not be hard to combine a 
similar analytic approach with attitudinal data covering aspects of shopper satisfaction to get a clear picture of the implications of deviation.

Beyond attitudinal measures, it would be interesting to obtain additional information about each shopper (e.g., demographics, media habits, behavior from other domains) and link them to the deviation measures. For instance, we may want to study how order and travel deviations differ across gender, age, and previous experience in the store. Another particularly interesting problem is whether the shopper indeed comes into the store with a shopping list in hand, and the extent to which they deviate from the list in their actual shopping decisions.

With additional data available in the future, our research can be extended in other directions as well. We briefly discuss some of these future research directions below:

(i) Natural experiments/field studies: In this paper, we explore deviation measures within a stable context for a particular grocery store. By performing similar analysis on several different grocery stores and thus conducting a cross-store study, we can study how store layout is related to order and travel deviations, and ultimately to sales volume and hence profitability. Alternatively, one can also perform a controlled field experiment, where the locations of products within the store are altered. Researchers can then analyze how the changes in product location affect the consumers' order and travel deviations, as well as on category sales. This can generate important insight for store design.

(ii) Incorporate shopping time: Researchers may not only be interested in deviation as measured by path distance, but also in terms of the time associated with each trip. An important dimension that we did not address in this paper is the amount of time that shoppers spend deliberating about their purchases, or aimlessly loitering, within a given zone. We can not address this issue with our cart-based RFID data because we do not observe the shopper's behavior directly, and 
without additional information about each consumer, it is difficult to control for individual-level speed differences. But as data collection technology further matures (e.g., using video recordings instead of - or in addition to - RFID tracking), this time dimension can fruitfully be explored.

(iii) Nonstationarilty: If we are able to obtain the identify of each shopper in the RFID data (e.g., by connecting the RFID data to a store loyalty card program), we can then obtain a panel data of shopping paths. By tracing each consumer over time, we can investigate whether order and travel deviation is a person-level trait or a trip-level trait. We can further study whether people tend to exhibit more or less departures from the TSP as they become more familiar with the store. This leads to a managerially relevant issue: can shoppers be "trained" to exhibit more or less order/travel deviations?

(iv) Online TSP: Another promising extension is to compare the observed behavior against some other optimality paradigm(s). In this research, the observed behavior is compared against the TSP, which is optimal under a strong set of assumptions, as we described. Although we have provided an initial look into the implications of relaxing some of these assumptions, the online TSP (Ausiello et al. 2001) would handle this task more comprehensively.

Beyond these specific, incremental changes to the analyses performed here, we believe that further exploring the relationships between grocery shopping behavior and formal optimization problems will lead to many interesting insights, both for academicians and practitioners, and will further complement our existing knowledge about the descriptive aspects of these paths. 


\section{References}

Adler, T., and M. Ben-Akiva (1979), “A Theoretical and Empirical Model of Trip Chaining Behavior," Transportation Research B, 13 (B), 243-257.

Ausiello, G., E. Feuerstein, S. Leonardi, L. Stougie, and M. Talamo (2001), “Algorithms for the on-line traveling salesman," Algorithmica, 29 (4), 560-581.

Bellmore, M., and G. L. Nemhauser (1968), "The Traveling Salesman Problem: A Survey," Operations Research, 16 (3), 538-558.

Burke, R. R. (1996), "Virtual Shopping: Breakthrough in Marketing Research,” Harvard Business Review, Mar-Apr, 120-131.

Camerer, C., F, T. Ho, and J. Chong (2004), “A Cognitive Hierarchy Model of Games,” Quarterly Journal of Economics, 861-898.

Chalasani, P., R. Motwani, and A. Rao (1996), “Algorithms for Robot Grasp and Delivery,” $2^{\text {nd }}$ International Workshop on Algorithmic Foundations of Robotics.

Cormen, T. H., C. E. Leiserson, R. L. Rivest, and C. Stein (2001), Introduction to Algorithms, $2^{\text {nd }}$ Edition. MIT Press.

Dellaert, B. G., T. A. Arentze, M. Bierlaire, A.W.J. Borgers, and H. J.P. Timmermans (1998), "Investigating Consumers' Tendency to Combine Multiple Shopping Purposes and Destinations," Journal of Marketing Research, 35 (2), 177-188.

Dogu, U., and F. Erkip (2000), "Spatial Factors Affecting Wayfinding and Orientation: A Case Study in a Shopping Mall," Environment and Behavior, 32 (6), 731-755.

Eilon, S., C. D. T. W. Gandy, and N. Christofides (1971), Distribution Management: Mathematical Modelling and Practical Analysis, Griffin, London.

Gilmore, P. C., and R. E. Gomory (1964), "Seqeuencing a One-State Variable Machine: A Solvable Case of the Traveling Salesman Problem," Operations Research, 12, 655-679.

Goffe, W. L., G. D. Ferrier, and J. Rogers (1994), “Global Optimization of Statistical Functions with Simulated Annealing," Journal of Econometrics, 60, 65-99.

Golledge, R. (1999), Wayfinding Behavior: Cognitive Mapping and Other Spatial Processes, John Hopkins University Press, Baltimore.

Guadagni, P. M., and J. D.C. Little (1983), “A Logit Model of Brand Choice Calibrated on Scanner Data," Marketing Science, 2(3), 203-238. 
Houser, D., M. Keane, and K. McCabe (2004), "Behavior in a Dynamic Decision Problem: An Analysis of Experimental Evidence Using a Bayesian Type Classification Algorithm," Econometrica, 72 (May), 781-822.

Hui, S. K., E. T. Bradlow, and P. S. Fader (2007a), “An Integrated Model of Shopping Paths and Purchase Behavior," Working Paper, available at http://ssrn.com/abstract=960960.

Hui, S. K., P. S. Fader, and E. T. Bradlow (2007b), "Path Data in Marketing: An Integrative Framework and Prospectus for Model-Building," Working Paper, available at http://ssrn.com/abstract=930141.

Hutchinson, J. W., and R. J. Meyer (1994), "Dynamic Decision Making: Optimal Policies and Actual Behavior in Sequential Choice Problems,” Marketing Letters, 5(4), 369-382.

Larson, J. S., E. T. Bradlow, and P. S. Fader (2005), “An Exploratory Look at Supermarket Shopping Paths," International Journal of Research in Marketing, 22 (4), 395-414.

Lawler, E. L. (1985), The Traveling Salesman Problem: A Guided Tour of Combinatorial Optimization. Wiley.

Lenstra, J. K., and A. H. G. Rinnooy Kan (1975), "Some Simple Applications of the Travelling Salesman Problem," Operational Research Quarterly, 26 (4), 717-733.

MacGregor, J. N., and T. Ormerod (1996), "Human Performance on the Traveling Salesman Problem," Perception and Psychophysics, 58, 527-539.

MacGregor, J.N., T.C. Ormerod, and E.P. Chronicle (1999), "Spatial and Contextual Factors in Human Performance on the Traveling Salesperson Problem," Perception, 28 (11), 14171427.

MacGregor, J.N, T.C. Ormerod, and E.P. Chronicle (2000), "Model of Human Performance on the Traveling Salesperson Problem," Memory and Cognition, 28 (7), 1183-1190.

Meyer, R. J., and J. Assuncao (1990), “The Optimality of Consumer Stockpiling Strategies," Marketing Science, 9(1), 18-41.

Neslin, S. A., and L. Greenhalgh (1983), "Nash's Theory of Cooperative Games as a Predictor of the Outcomes of Buyer-Seller Negotiations: An Experiment in Media Purchasing," Journal of Marketing Research, 20 (4), 368-379.

Polivanova, N. I. (1974), “[Functional and Structural Aspects of the Visual Components of Intuition in Problem Solving," Voprosy Psikhologii, 4, 41-51.

Schelhorn, T., D. Sullivan, M. Haklay, and M. Thurstain-Goodwin (1999), "Streets: An AgentBased Pedestrain Model," Center for Advanced Spatial Analysis, Working Paper. 
Seale, D. A., and A. Rapoport (2000), "Optimal Stopping Behavior with Relative Ranks: The Secretary Problem with Unknown Population Size," Journal of Behavioral Decision Making, 13(4), 391-411.

Sorensen, H. (2003), “The Science of Shopping,” Marketing Research, 15(3), 30-35.

Thill, J. C. and I. Thomas (1991), "Towards Conceptualizing Trip-Chaining Behavior: A Review," Geographical Analysis, 19 (1), 1-17.

Titus, P. A., and P. B. Everett (1996), “Consumer Wayfinding Tasks, Strategies, and Errors: An Exploratory Field Study,” Psychology and Marketing, 13 (3), 265-290.

Vickers, D., M. Butavicius, M. Lee, and A. Medvedev (2001), "Human Performance on Visually Presented Traveling Salesman Problem," Psychological Research, 65, 34-45. 


\section{Appendix 1: Applying the TSP algorithm to grocery shopping paths}

As discussed in Section 3, we use two algorithms to solve for the optimal solution: (i) exhaustive search, and (ii) simulated annealing. Exhaustive search is used when the number of purchase location is less than or equal to 10 ; simulated annealing is used when the number of purchase location is more than 11.

\section{$\underline{\text { Algorithm I: Exhaustive Search }}$}

We generate every possible permutation of the order of purchase locations then compute the total distance associated with each order. The optimal solution is the order that results in the shortest total distance. Table 3 of the text gives an example in the case of 3 purchase locations.

\section{Algorithm II: Simulated Annealing}

When the number of purchase locations is greater than 10 , the number of possible permutations is too large to conduct an exhaustive search. In this case, we use simulated annealing to obtain the optimal solution. Our implementation here is based on the three-step algorithm of Goffe (1994). Readers are referred to Goffe (1994) for a full discussion of the theoretical foundations of the simulated annealing algorithm.

Step I: First, we define the "neighborhood" of an order. We define a "move" to be a pairwise switch of two locations. That is, ADCB can be reached from ABCD in one move (i.e., only B-D are switched).

Step II: Then, we randomly choose a starting order. Each order has an equal probability of being chosen as the initial order.

Step III: We specify a "starting temperature" $T_{0}$ and a "cooling schedule" $T_{n}$ (the temperature at step $n) . T_{0}$ is defined so that initially, around $80 \%$ of the "uphill" moves (i.e., moves that lead to a 
large total travel distance) will be accepted, as recommended by Goffe (1994). We use an exponentially cooling schedule for $T_{n}$, which takes the following form:

$$
T_{n}=T_{0} k^{n}
$$

where $k<1$ is a tuning constant that controls how fast the temperature is cooled. In our case, $k$ is chosen to be at least 0.99999 to ensure that the algorithm has a high probability of reaching the global optimal solution.

Step IV: We then start the annealing algorithm at the starting temperature, and loop until $T_{n}$ falls below a small constant $(0.001)$. At each iteration $n$, the following steps are performed:

(i) A "proposal" order, denoted as $C^{*}$, is randomly generated from the neighborhood of the current order, denoted as $C$.

(ii) The total distance associated with the proposal order, $D^{*}$, is calculated.

(iii) $D^{*}$ is compared to the total distance associated with the current order, $D$, if $D^{*}<D$, then $C$ is replaced by $C^{*}$. Otherwise, $\mathrm{C}$ is replaced by $\mathrm{C}^{*}$ with probability $\exp \left(\left(D-D^{*}\right) / T_{n}\right)$.

\section{Appendix 2: PathTracker ${ }^{\circledR}$ data preparation and cleaning procedure (Hui et al. 2007a)}

A PathTracker ${ }^{\circledR}$ dataset consists of a large number of "trips" that include both the shopping paths and purchase data at the checkout counter. Each trip starts when the shopping cart is taken at the store entrance and ends when the cart is pushed through the checkout line to the other side of the checkout counter. A shopping path is represented by a list of $(x, y)$ coordinates at five second intervals (a "blink") that indicate the current location of the shopping cart. Purchase information are collected in the form of scanner data, indicating which products, represented by their UPCs, are purchased. Within the PathTracker ${ }^{\circledR}$ system, each location within the store is represented by a pair of $(x, y)$ coordinates; together with the scanner data, we can map 
each purchase back to the store location where it was made. Sometimes, a product category can be located in more than one area within the store. In this case, we need to rely on simple heuristics or statistical models to determine (or infer) which store location is associated with the purchase. In this research, we assign a purchase to the feasible location where the shopper spent the longest time shopping. This assignment heuristic is based on the intuition that buying a product should take more time than just walking past an area. While we recognize that this assignment algorithm is imperfect, we believe that it provides a good starting point for our initial study on shoppers' behavior.

To prepare our data for analysis, we first screen out paths that contain purchases in only one category, because we are interested in determining whether a consumer's order of purchases is optimal. After eliminating these paths (roughly $25 \%$ of all paths), we have a total of 993 paths for analysis. Next, to facilitate our analysis, we discretize the store into 96 zones based on our discussion with Sorensen Associates. The discretization is shown in Figure 3.

At first sight, the procedure of discretization appears to "throw away" some of the data, since the variation of the data at a resolution finer than zones is lost. But this procedure leads to three main advantages: (1) It simplifies modeling by limiting the number of possible locations in the store; we can then represent the shopping path as a series of finite choice problems, which allows us to more efficiently solve for the TSP-optimal path (Hui et al. 2007b), as explained in Section 4 ; (2) the location of the shopping cart is not a perfect proxy for the shopper: the shopper can "park" his cart at a certain spot and then shop somewhere else. Thus, it is more reasonable to assume that the location of the cart gives us some indication to the general region where the shopper is located, rather than treating it as the shopper's exact location. (3) Similarly, we only know the general position of each product in the store, up to a certain degree of error. Thus, the 
procedure of discretization brings our analysis closer to the resolution of the measurement accuracy of our data. Third, if data loss is indeed an issue, regions can be made smaller to increase the resolution of the discretization. The amount of data loss can then be minimized. Thus, the framework developed here is fully general.

After discretizing the store, we implicitly take into account the existence of physical barriers (e.g. aisles, walls) in the store by representing the store as a "graph": a mathematical object defined by "nodes" that represent regions and "edges" that depicts the connectivity between different regions. A node is placed at the center of each zone. An edge is drawn between two nodes if they represent two adjacent regions, indicating that it is possible to move from one to the other without going through any other node. Figure 4 shows how the grocery store is represented as a graph of 96 nodes, referring to each of the 96 zones.

For example, although node A and node B are close to each other in Euclidean distance (they are in adjacent aisles), one would have to go through at least 6 intermediate nodes to go from A to B. This is due to the absence of an edge connecting node A and node B. The shortest travel distance between any pairs of locations in the store can be approximated by the distance of the shortest path connecting their respective nodes. Thus, the graph is a faithful representation of the distances between each zone in the grocery store, since it takes into account the multiple spatial constraints of the store.

\section{Appendix 3: Procedure of estimating fractional look-ahead}

We estimate the optimality index for each shopper based on the following procedure: At step i, based on the shopper's current location and the purchases that still need to be made, the 1-step look ahead rule will recommend a certain location to visit. 
(ii) The shopper has probability $p$ of following the 1-step look ahead rule, and probability 1-p of randomly choosing his next location to visit. If a random algorithm is used, we assume that the person chooses uniformly among each of the $n_{i}$ possible alternatives, i.e., each with probability $1 / n_{i}$. The number of alternatives is defined by the geometry of the grocery store, which we discussed in Section 3, and is shown in Figure 3 and 4.

(iii) Thus, the overall probability of traveling to the location suggested by 1-step look ahead is $p_{i}=p+\frac{1-p}{n_{i}}$.

(iv) The overall likelihood of the shopper's order of transversal is thus $\prod_{i} p_{i}^{z_{i}}\left(1-p_{i}\right)^{1-z_{i}}$, where $z_{i}$ equals 1 if the 1 -step look ahead rule is followed at step $i$, and 0 otherwise.

(v) Finally, $p$ is estimated for each shopper using maximum likelihood estimation. 


\begin{tabular}{|l|l|l|r|r|r|}
\hline & $\begin{array}{l}\text { Distance from } \\
\text { Entrance }\end{array}$ & $\begin{array}{l}\text { Distance from } \\
\text { A }\end{array}$ & $\begin{array}{l}\text { Distance from } \\
\text { B }\end{array}$ & $\begin{array}{l}\text { Distance from } \\
\text { C }\end{array}$ & $\begin{array}{l}\text { Distance to } \\
\text { checkout }\end{array}$ \\
\hline $\mathrm{A}$ & 8.31 & 0.00 & 1.59 & 6.55 & 9.72 \\
\hline $\mathrm{B}$ & 8.06 & 1.59 & 0.00 & 5.41 & 8.16 \\
\hline $\mathrm{C}$ & 4.91 & 6.55 & 5.41 & 0.00 & 5.28 \\
\hline
\end{tabular}

Table 1. Distances between entrance, checkout, A, B, and C (C is selected).

\begin{tabular}{|l|l|l|r|}
\hline & $\begin{array}{l}\text { Entrance to First } \\
\text { location }\end{array}$ & $\begin{array}{l}\text { First location to } \\
\text { Second location }\end{array}$ & $\begin{array}{l}\text { Total distance for first } \\
\text { two moves }\end{array}$ \\
\hline AB & 8.31 & 1.59 & 9.90 \\
\hline AC & 8.31 & 6.55 & 14.86 \\
\hline BA & 8.06 & 1.59 & 9.65 \\
\hline BC & 8.06 & 5.41 & 13.47 \\
\hline CA & 4.91 & 6.55 & 11.46 \\
\hline CB & 4.91 & 5.41 & 10.32 \\
\hline
\end{tabular}

Table 2. Illustration of 2-step look ahead algorithm (BA is selected).

\begin{tabular}{|l|r|l|l|l|r|}
\hline & $\begin{array}{l}\text { Entrance to } \\
\text { first location }\end{array}$ & $\begin{array}{l}\text { First location } \\
\text { to second } \\
\text { location }\end{array}$ & $\begin{array}{l}\text { Second } \\
\text { location to } \\
\text { third location }\end{array}$ & $\begin{array}{l}\text { Third } \\
\text { location to } \\
\text { checkout }\end{array}$ & $\begin{array}{l}\text { Total Trip } \\
\text { Distance }\end{array}$ \\
\hline ABC [4] & 8.31 & 1.59 & 5.41 & 5.28 & 20.59 \\
\hline ACB & 8.31 & 6.55 & 5.41 & 8.16 & 28.43 \\
\hline BAC [2] & 8.06 & 1.59 & 6.55 & 5.28 & 21.48 \\
\hline BCA & 8.06 & 5.41 & 6.55 & 9.72 & 29.74 \\
\hline CAB [3] & 4.91 & 6.55 & 1.59 & 8.16 & 21.21 \\
\hline CBA [1] & 4.91 & 5.41 & 1.59 & 9.72 & 21.63 \\
\hline
\end{tabular}

Table 3. Illustration of 4-step (TSP-Optimal) look ahead. ([4]: Optimal under $k=4$; [3]: Optimal under $k=3$; [2]: Optimal under $k=2$; [1]: Optimal under $k=1$ ).

\begin{tabular}{|l|r|r|r|r|}
\hline & \multicolumn{1}{|c|}{ Mean } & \multicolumn{1}{|c|}{ S.D. } & \multicolumn{1}{|c|}{ Min } & \multicolumn{1}{c|}{ Max } \\
\hline $\begin{array}{l}\text { Number of product categories } \\
\text { purchased }\end{array}$ & 7.1 & 4.0 & 2.0 & 25.0 \\
\hline Total travel distance (in feet) & 2513.0 & 1193.4 & 233.9 & 11234.4 \\
\hline $\begin{array}{l}\text { Number of unique zones } \\
\text { visited }\end{array}$ & 49.9 & 14.2 & 5.0 & 83.0 \\
\hline $\begin{array}{l}\text { Number of unique aisles } \\
\text { entered }\end{array}$ & 7.3 & 3.4 & 0.0 & 15.0 \\
\hline $\begin{array}{l}\text { Number of unique aisles } \\
\text { transversed }\end{array}$ & 2.6 & 1.7 & 0.0 & 9.0 \\
\hline
\end{tabular}

Table 4. Key summary statistics of the PathTracker ${ }^{(B)}$ dataset. 


\begin{tabular}{|l|r|}
\hline Category & $\begin{array}{l}\text { Proportion of shoppers who purchase } \\
\text { the product category }\end{array}$ \\
\hline Fruits & $55.3 \%$ \\
\hline Vegetables & $52.2 \%$ \\
\hline Butter/Cheese/Cream & $40.0 \%$ \\
\hline Carbonated Beverages & $25.4 \%$ \\
\hline Salty Snacks & $24.4 \%$ \\
\hline Cookies and Crackers & $23.9 \%$ \\
\hline Milk & $23.7 \%$ \\
\hline Ice cream & $20.7 \%$ \\
\hline Loaf Bread & $20.5 \%$ \\
\hline Cereal (Ready-to-eat) & $18.1 \%$ \\
\hline
\end{tabular}

Table 5. Top 10 categories purchased.

\begin{tabular}{|l|r|r|r|r|r|}
\hline & \multicolumn{1}{|c|}{ Mean } & \multicolumn{1}{|c|}{ S.D. } & \multicolumn{1}{|c|}{ Mean \% } & \multicolumn{1}{|c|}{ Min \% } & \multicolumn{1}{c|}{ Max \% } \\
\hline Optimal Path & 612.0 & 189.4 & $27.5 \%$ & $5.4 \%$ & $94.7 \%$ \\
\hline Order Deviation & 89.5 & 107.9 & $3.1 \%$ & $0.0 \%$ & $17.1 \%$ \\
\hline Travel Deviation & 1811.5 & 1021.6 & $69.4 \%$ & $5.3 \%$ & $94.6 \%$ \\
\hline Total Distance & 2513.0 & 1193.4 & & & \\
\hline
\end{tabular}

Table 6. Summary statistics from decomposition analysis. 


\begin{tabular}{|c|c|c|c|c|}
\hline $\begin{array}{l}\text { Order Deviation }(\mathrm{H} / \mathrm{L}) \\
\text { Travel Deviation }(\mathrm{H} / \mathrm{L})\end{array}$ & $\begin{array}{c}\text { Group } 1 \\
\\
\text { L } \\
\text { L }\end{array}$ & $\begin{array}{c}\text { Group } 2 \\
\\
\text { H } \\
\text { L }\end{array}$ & $\begin{array}{c}\text { Group } 3 \\
\text { L } \\
\text { H }\end{array}$ & $\begin{array}{c}\text { Group } 4 \\
\\
\mathrm{H} \\
\mathrm{H}\end{array}$ \\
\hline Number of shoppers & 203 & 294 & 294 & 202 \\
\hline Mean $\%$ order deviation & $0.4 \%$ & $6.3 \%$ & $0.6 \%$ & $4.8 \%$ \\
\hline Mean $\%$ travel deviation & $59.5 \%$ & $62.5 \%$ & $78.6 \%$ & $76.1 \%$ \\
\hline $\begin{array}{l}\text { Mean unique number of } \\
\text { zones visited }\end{array}$ & 38.2 & 52.1 & 48.9 & 59.7 \\
\hline $\begin{array}{l}\text { Mean basket size } \\
\text { (number of categories) }\end{array}$ & 4.5 & 8.7 & 5.6 & 9.6 \\
\hline $\begin{array}{l}\text { Mean unique number of } \\
\text { aisles entered }\end{array}$ & 4.7 & 7.7 & 7.1 & $\overline{9.6}$ \\
\hline $\begin{array}{l}\text { Mean unique number of } \\
\text { aisles transversed }\end{array}$ & 1.4 & 2.8 & 2.5 & 3.7 \\
\hline
\end{tabular}

Table 7. Summary statistics of the clusters of shoppers (H: high; L: low). 


\begin{tabular}{|c|c|c|c|c|c|}
\hline \multicolumn{6}{|c|}{ Categories overrepresented in Group $1(p<.05)$} \\
\hline Category & Group 1 & Group 2 & Group 3 & Group 4 & Overall \\
\hline Butter-Cheese-Cream & $7.4 \%$ & $5.9 \%$ & $5.1 \%$ & $4.9 \%$ & $5.6 \%$ \\
\hline Milk & $5.4 \%$ & $3.5 \%$ & $3.1 \%$ & $2.4 \%$ & $3.3 \%$ \\
\hline $\begin{array}{l}\text { Meat-Poulty-Seafood Manufactured } \\
\text { Prepack }\end{array}$ & $2.6 \%$ & $1.3 \%$ & $1.2 \%$ & $1.7 \%$ & $1.5 \%$ \\
\hline Prepackaged Deli Prepared Lunch & $0.4 \%$ & $0.2 \%$ & $0.0 \%$ & $0.2 \%$ & $0.2 \%$ \\
\hline Fruits & $10.9 \%$ & $6.4 \%$ & $9.7 \%$ & $6.5 \%$ & $7.8 \%$ \\
\hline Vegetables & $9.8 \%$ & $5.8 \%$ & $10.1 \%$ & $6.0 \%$ & $7.4 \%$ \\
\hline Tobacco & $1.3 \%$ & $0.4 \%$ & $0.8 \%$ & $0.5 \%$ & $0.7 \%$ \\
\hline \multicolumn{6}{|c|}{ Categories overrepresented in Group $2(p<.05)$} \\
\hline Category & Group 1 & Group 2 & Group 3 & Group 4 & Overall \\
\hline Pudding/Dry Dessert & $0.3 \%$ & $0.5 \%$ & $0.1 \%$ & $0.3 \%$ & $0.3 \%$ \\
\hline Tea & $0.0 \%$ & $0.2 \%$ & $0.0 \%$ & $0.2 \%$ & $0.1 \%$ \\
\hline Canned Meat & $0.2 \%$ & $0.4 \%$ & $0.0 \%$ & $0.1 \%$ & $0.2 \%$ \\
\hline Pasta & $0.5 \%$ & $1.3 \%$ & $0.8 \%$ & $1.0 \%$ & $1.0 \%$ \\
\hline Paper Towels & $0.5 \%$ & $0.9 \%$ & $0.7 \%$ & $0.4 \%$ & $0.7 \%$ \\
\hline Prepared Food/Dry Dinner & $0.3 \%$ & $1.4 \%$ & $0.9 \%$ & $1.3 \%$ & $1.1 \%$ \\
\hline \multicolumn{6}{|c|}{ Categories overrepresented in Group $3(p<.05)$} \\
\hline Candy/Gum/Mint & $2.6 \%$ & $2.6 \%$ & $3.3 \%$ & $1.9 \%$ & $2.6 \%$ \\
\hline Fruits & $10.9 \%$ & $6.4 \%$ & $9.7 \%$ & $6.5 \%$ & $7.8 \%$ \\
\hline Vegetables & $9.8 \%$ & $5.8 \%$ & $10.1 \%$ & $6.0 \%$ & $7.4 \%$ \\
\hline \multicolumn{6}{|c|}{ Categories overrepresented in Group $4(p<.05)$} \\
\hline Baby Food & $0.2 \%$ & $0.0 \%$ & $0.2 \%$ & $0.5 \%$ & $0.2 \%$ \\
\hline Bagels/Breadsticks & $0.3 \%$ & $0.8 \%$ & $0.6 \%$ & $1.1 \%$ & $0.8 \%$ \\
\hline Bottled Water & $0.9 \%$ & $0.7 \%$ & $1.0 \%$ & $1.8 \%$ & $1.1 \%$ \\
\hline Coffee & $0.2 \%$ & $0.7 \%$ & $0.3 \%$ & $1.0 \%$ & $0.6 \%$ \\
\hline Cookies and Crackers & $3.0 \%$ & $3.4 \%$ & $2.6 \%$ & $4.1 \%$ & $3.4 \%$ \\
\hline Frozen Pizza/Snacks & $1.0 \%$ & $1.1 \%$ & $1.2 \%$ & $2.1 \%$ & $1.4 \%$ \\
\hline Household Cleaners & $0.4 \%$ & $0.6 \%$ & $0.4 \%$ & $1.0 \%$ & $0.7 \%$ \\
\hline
\end{tabular}

Table 8. Comparison of product category purchase for each group, controlling for basket size. 


\begin{tabular}{|c|c|c|}
\hline & $\begin{array}{l}\text { Group A } \\
(k \geq 1)\end{array}$ & $\begin{array}{l}\text { Group B } \\
(k<1)\end{array}$ \\
\hline Number of shoppers & 489 & 504 \\
\hline Mean $\%$ order deviation & $1.0 \%$ & $5.1 \%$ \\
\hline Mean \% travel deviation & $69.6 \%$ & $69.3 \%$ \\
\hline Mean unique number of zones visited & 44.3 & 55.3 \\
\hline Mean basket size (number of categories) & 5.5 & 8.6 \\
\hline Mean unique number of aisles entered & 6.0 & 8.5 \\
\hline Mean unique number of aisles transversed & 2.0 & 3.1 \\
\hline
\end{tabular}

Table 9. Summary statistics of the two groups segmented by $k$.

\begin{tabular}{|c|c|c|c|}
\hline \multicolumn{4}{|c|}{ Categories Overrepresented in High-k group (group A) } \\
\hline Category & Group A & Group B & Overall \\
\hline Fruits & $10.5 \%$ & $6.1 \%$ & $7.8 \%$ \\
\hline Vegetables & $10.0 \%$ & $5.7 \%$ & $7.4 \%$ \\
\hline Tobacco & $1.0 \%$ & $0.4 \%$ & $0.7 \%$ \\
\hline \multicolumn{4}{|c|}{ Categories Overrepresented in Low-k group (group B) } \\
\hline Category & Group A & Group B & Overall \\
\hline Bagels/Breadsticks & $0.6 \%$ & $0.9 \%$ & $0.8 \%$ \\
\hline Spice/Seasonings & $0.4 \%$ & $0.9 \%$ & $0.7 \%$ \\
\hline Cereal (Ready to Eat) & $2.0 \%$ & $2.9 \%$ & $2.6 \%$ \\
\hline Canned Dry Fruit & $1.2 \%$ & $1.8 \%$ & $1.6 \%$ \\
\hline Canned Meat Entrée & $0.0 \%$ & $0.4 \%$ & $0.3 \%$ \\
\hline Pasta & $0.6 \%$ & $1.3 \%$ & $1.0 \%$ \\
\hline Frozen Baked Goods & $0.4 \%$ & $0.9 \%$ & $0.7 \%$ \\
\hline Frozen Meat/Poultry/Seafood & $0.6 \%$ & $1.3 \%$ & $1.1 \%$ \\
\hline Paper Towels & $0.4 \%$ & $0.8 \%$ & $0.7 \%$ \\
\hline Prepared Food/Dry Dinner & $0.6 \%$ & $1.4 \%$ & $1.1 \%$ \\
\hline
\end{tabular}

Table 10. Comparison of category purchase between the low- $k$ group and high- $k$ group $(\mathrm{p}<.05)$. 


\begin{tabular}{|l|l|l|l|l|}
\hline Unplanned \% & TSP-Optimal \% & Order \% & Travel \% & Average $k$ \\
\hline 0 & $27.5 \%$ & $3.1 \%$ & $69.4 \%$ & 1.36 \\
\hline 10 & $27.3 \%$ & $3.0 \%$ & $69.7 \%$ & 1.37 \\
\hline 20 & $26.4 \%$ & $2.6 \%$ & $71.0 \%$ & 1.40 \\
\hline 30 & $25.5 \%$ & $2.3 \%$ & $72.2 \%$ & 1.35 \\
\hline 40 & $24.0 \%$ & $1.8 \%$ & $74.2 \%$ & 1.33 \\
\hline 50 & $22.1 \%$ & $1.3 \%$ & $76.6 \%$ & 1.27 \\
\hline
\end{tabular}

Table 11. Results of the sensitivity analysis regarding unplanned purchases.

\begin{tabular}{|l|l|l|l|l|}
\hline$m$ & TSP-Optimal \% & Order \% & Travel \% & Average $k$ \\
\hline 0 & $27.5 \%$ & $3.1 \%$ & $69.4 \%$ & 1.36 \\
\hline 1 & $29.8 \%$ & $3.8 \%$ & $66.4 \%$ & 1.37 \\
\hline 2 & $31.7 \%$ & $4.3 \%$ & $64.0 \%$ & 1.35 \\
\hline 3 & $33.2 \%$ & $5.2 \%$ & $61.6 \%$ & 1.24 \\
\hline 4 & $34.8 \%$ & $5.8 \%$ & $59.5 \%$ & 1.26 \\
\hline 5 & $36.1 \%$ & $5.9 \%$ & $58.0 \%$ & 1.30 \\
\hline
\end{tabular}

Table 12. Results of the sensitivity analysis regarding planned yet unpurchased categories. 


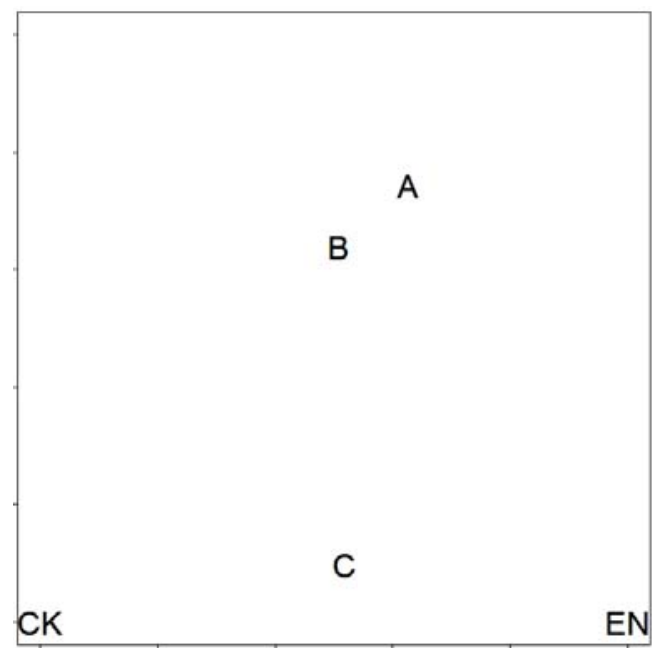

Figure 1. Example of $k$-step look-ahead algorithm. "EN": Entrance; "CK": Checkout.

Min distance for each path
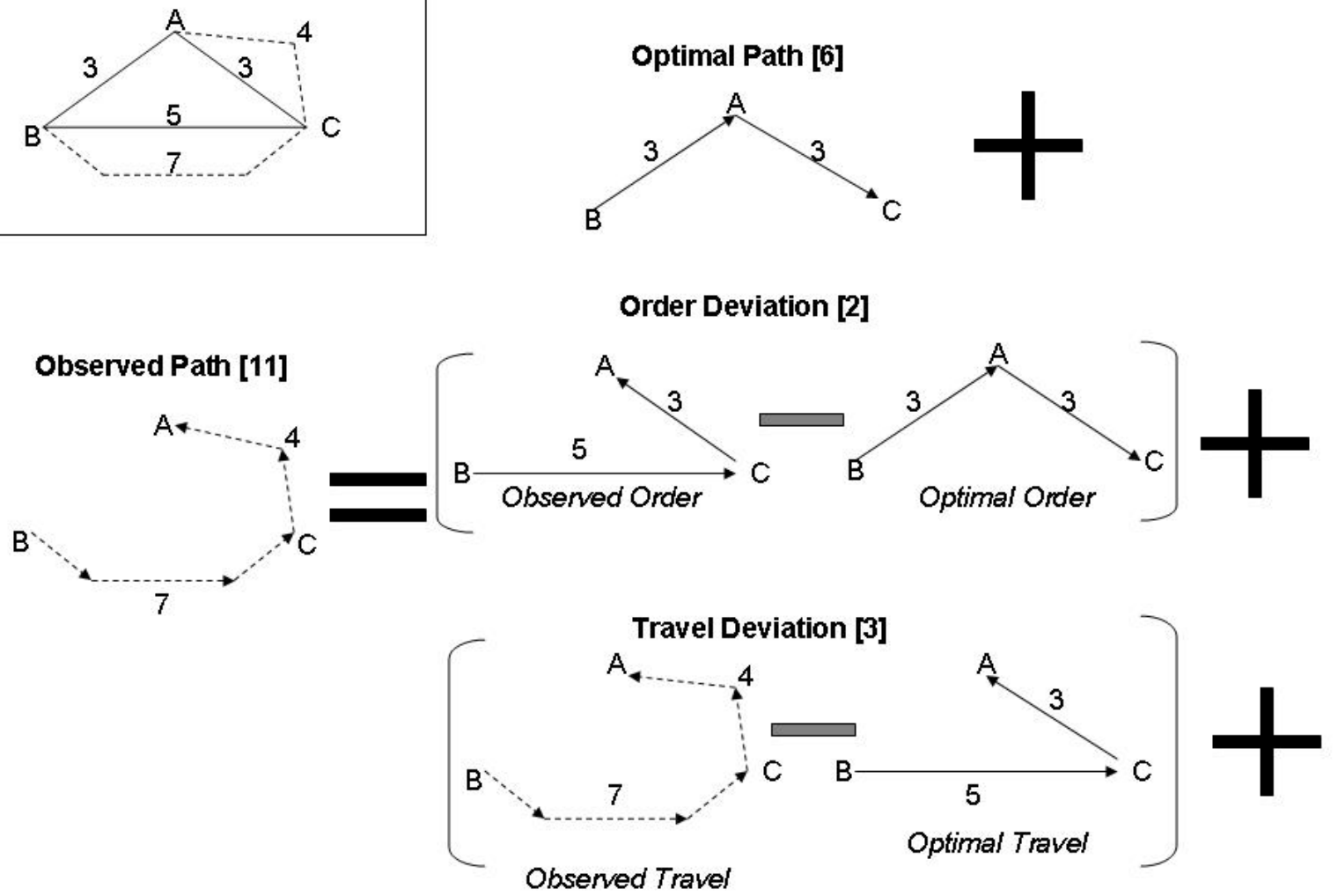

Figure 2. Deviation decomposition 


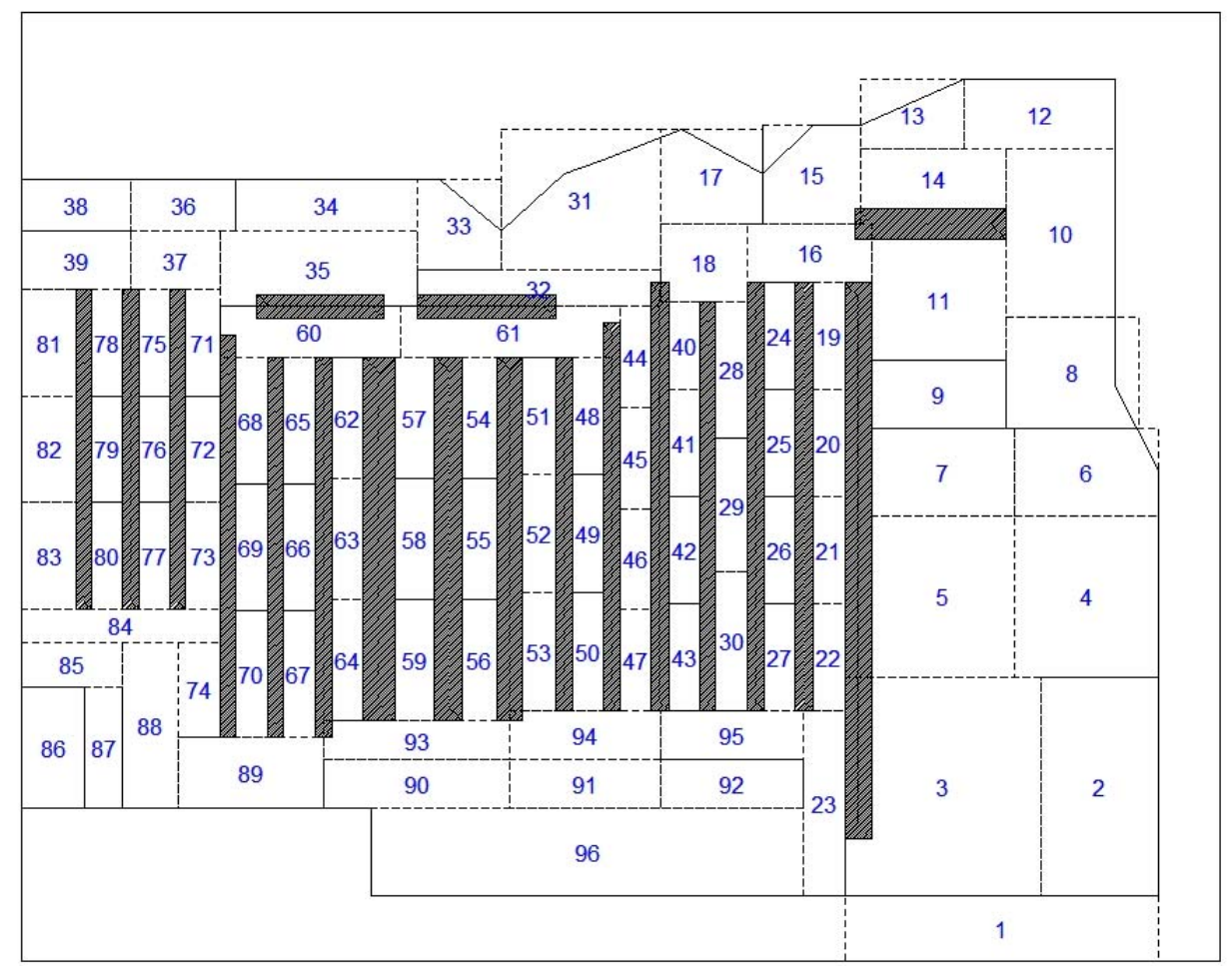

Figure 3. Grocery store divided into 96 zones

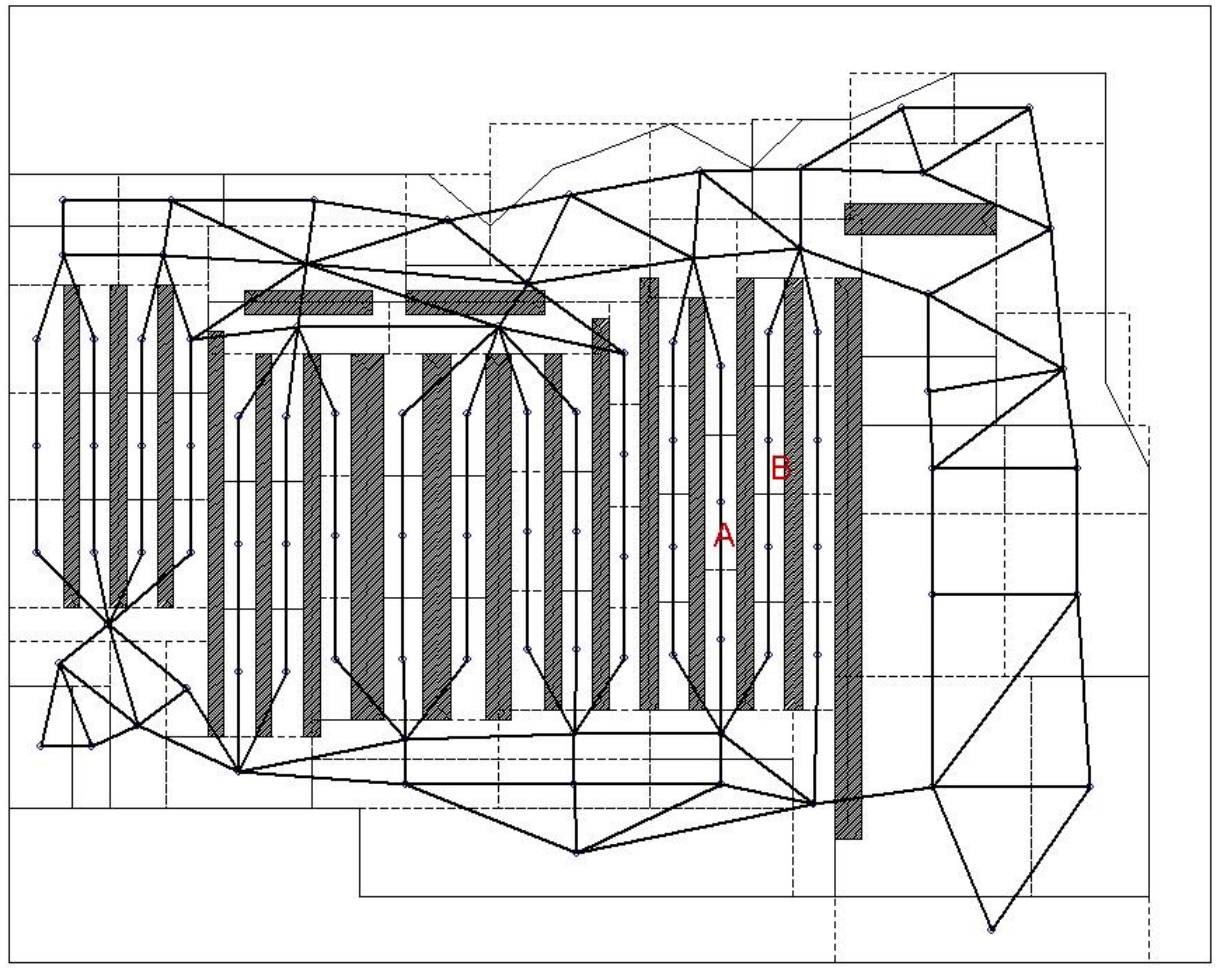

Figure 4. Grocery store represented by a graph of 96 nodes 


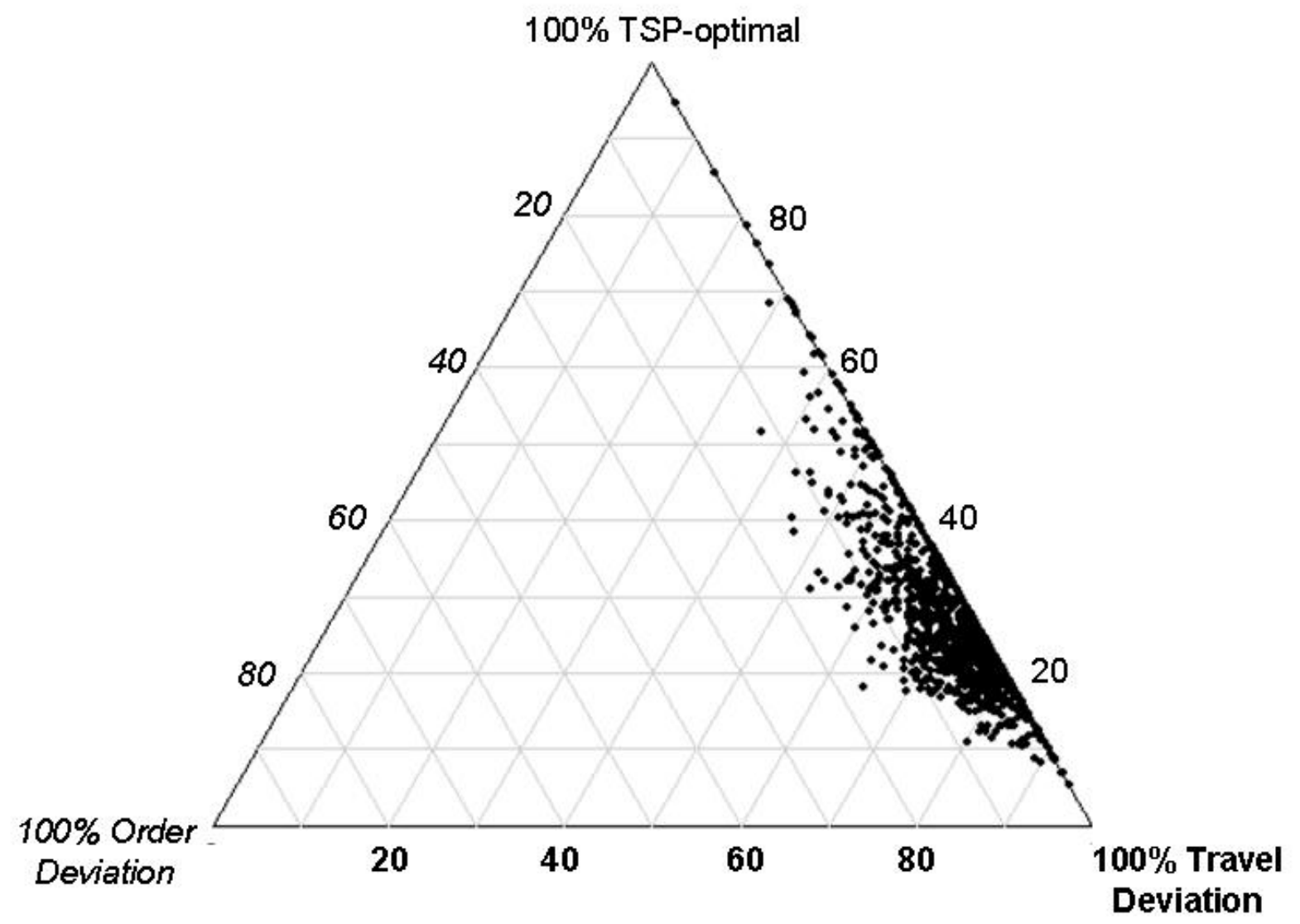

Figure 5. Triangle plot for optimal path, order deviation, and travel deviation. The different fonts and angled hashmarks indicate which scale corresponds to each dimension. 


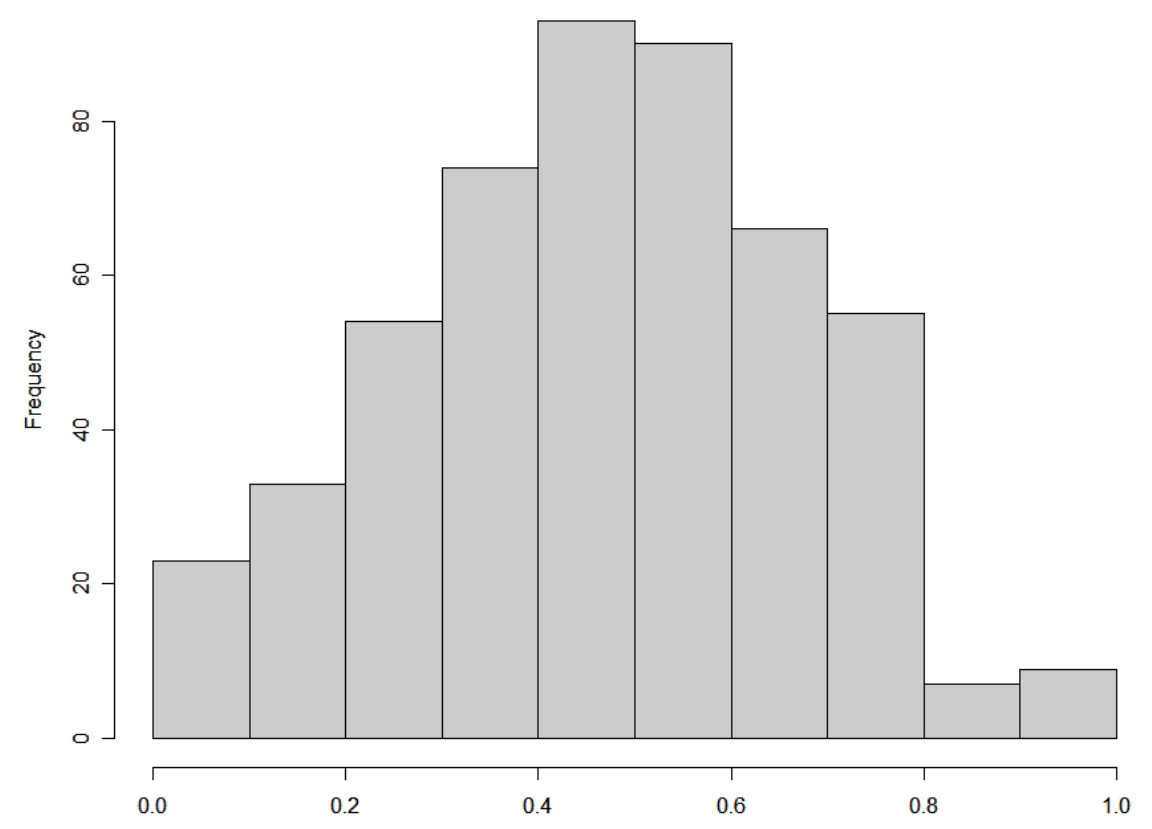

Figure 6. Distribution of $k$ for shoppers in group B.

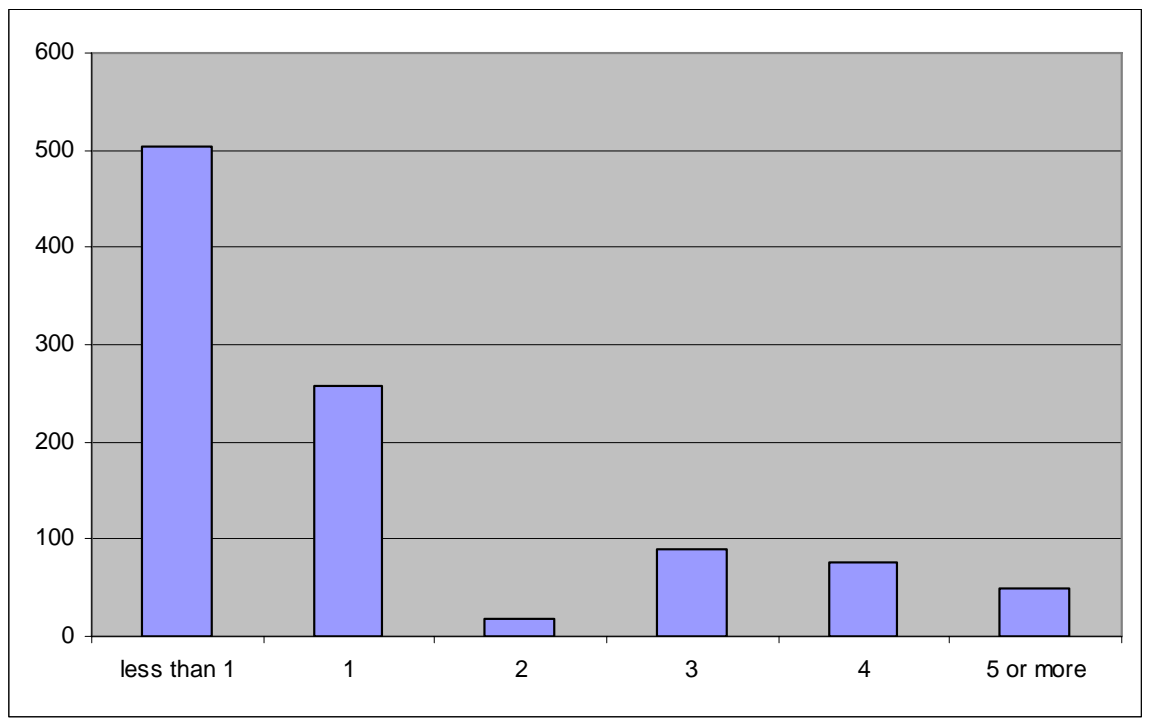

Figure 7. Distribution of $k$ for all shoppers. 